\title{
Polygon Number Algorithm for Peak-to-Average Ratio Reduction of Massive 5G Systems Using Modified Partial Transmit Sequence Scheme
}

\author{
Hayder Alkatrani, ${ }^{1}$ Muhammad Ilyas $\mathbb{D}^{2},{ }^{2}$ Salam Alyassri, ${ }^{1}$ Ali Nahar, ${ }^{3}$ Fadi Al-Turjman $\left(\mathbb{D},{ }^{4}\right.$ \\ Jawad Rasheed $\mathbb{D}^{5}{ }^{5}$ Ali Alshahrani, ${ }^{6}$ and Basil Al-Kasasbeh ${ }^{6}$ \\ ${ }^{1}$ Electrical \& Computer Engineering Department, University of Altinbas, Istanbul, Turkey \\ ${ }^{2}$ Electrical \& Electronics Engineering Department, University of Altinbas, Istanbul, Turkey \\ ${ }^{3}$ Electrical Engineering Department, University of Technology, Iraq \\ ${ }^{4}$ Artificial Intelligence Engineering Department, Research Center for AI and IoT, Near East University, Nicosia, Mersin 10, Turkey \\ ${ }^{5}$ Department of Computer Engineering, Istanbul Aydin University, Istanbul, Turkey \\ ${ }^{6}$ Arab Open University, Riyadh, Saudi Arabia
}

Correspondence should be addressed to Jawad Rasheed; jawadrasheed@aydin.edu.tr

Received 27 August 2021; Accepted 3 November 2021; Published 25 November 2021

Academic Editor: Deepak Gupta

Copyright (c) 2021 Hayder Alkatrani et al. This is an open access article distributed under the Creative Commons Attribution License, which permits unrestricted use, distribution, and reproduction in any medium, provided the original work is properly cited.

\begin{abstract}
The high peak-to-average power ratio (PAPR) of the transmit signal is a major shortcoming of OFDM systems, which results in band radiation and distortion due to the nonlinearity of the high-power amplifier (H.P). To resolve the traditional OFDM highPAPR issue, where the transmit sequence is designed to avoid similar data from being sent in the same order to reduce PAPR, there are numerous conventional ways for lowering the PAPR for OFDM system, such as selective mapping, tone reservation, block coding, filtering, clipping, and partial transmit sequence (PTS). This study proposes a new method called polygon number algorithm (PN) with conventional partial transmit sequence (C-PTS). This method (PN-PTS) processes the entered data before sending it, taking advantage of the number nonsimilarity according to the geometry of the number to prevent direct sending of similar data via PTS, and thus, this improved the level of PAPR rise in the proposed system. The amount of reduction that can be achieved in PAPR is up to $8 \mathrm{~dB}$ by different techniques. The best result obtained was the amount of reduction between the conventional method and the proposed method is 4.5683 where $N=64$. Besides this, there is no transmission of side information (SI), which improves transmission efficiency. Finally, this method is easy in the calculation process and the ciphering and deciphering of data, which adds a few calculations.
\end{abstract}

\section{Introduction}

The orthogonal frequency division multiplexing (OFDM) is a frequency division scheme in which the carrier frequencies are very close yet do not interfere with each other. The frequency spacing that is used in OFDM is very small, and its symbol period is bigger than or equal to the reciprocation of this spacing; OFDM usually has a high symbol duration, which reduces intersymbol interference (ISI) [1]. Multimedia communication systems necessitate a high data rate, which necessitates a large amount of transmission power and bandwidth. As a result, high compression efficiency is required to transmit wireless multimedia information over a limited available bandwidth [2]. A common problem with wireless communications is especially in a multipath environment so that the ISI prevents high-speed communication. Therefore, OFDM allows high-productivity communication by increasing the number of carrier frequencies and maintaining high spectral efficiency. Nonetheless, it has challenges to implementation. OFDM signals have trouble with high peak-to-average power ratio (PAPR), to convert digital-to-analog (DAC) signal with high PAPR 


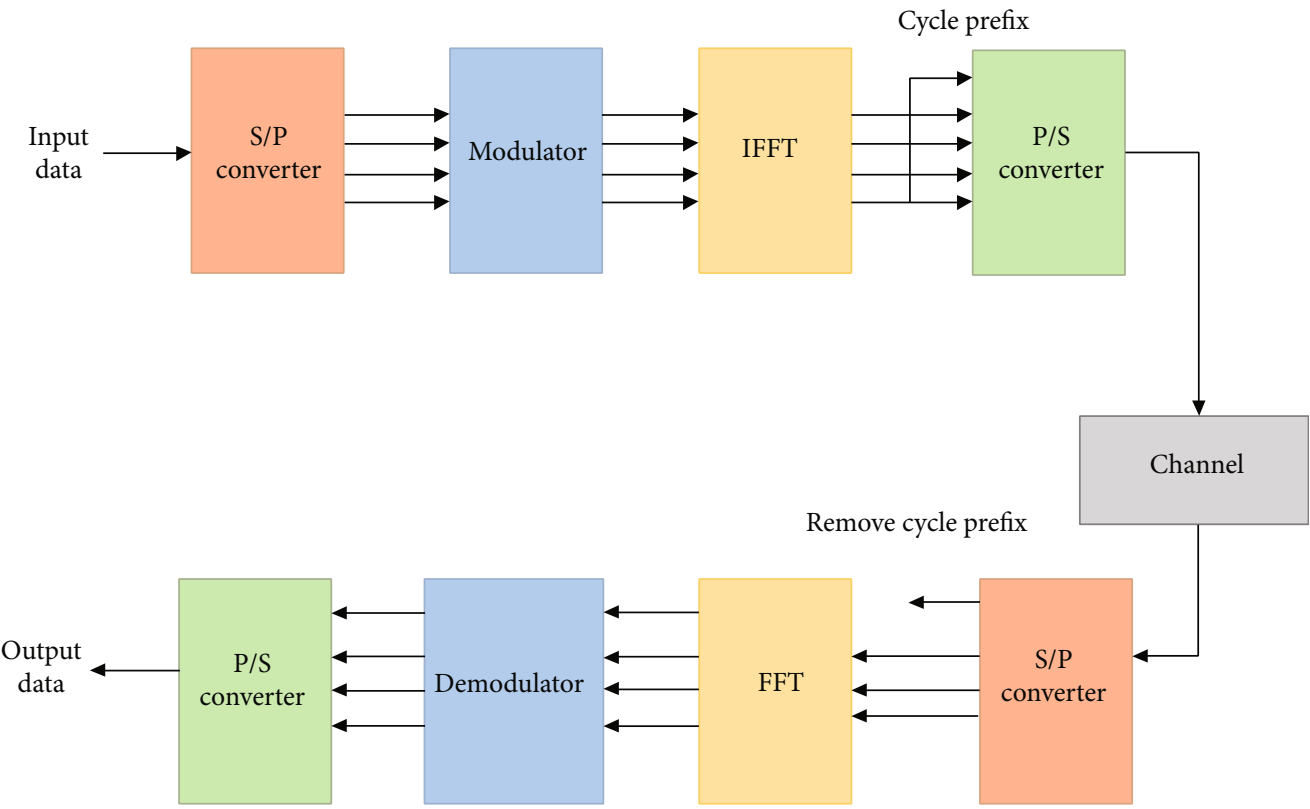

FIgURE 1: OFDM block diagram transmitter and receiver.

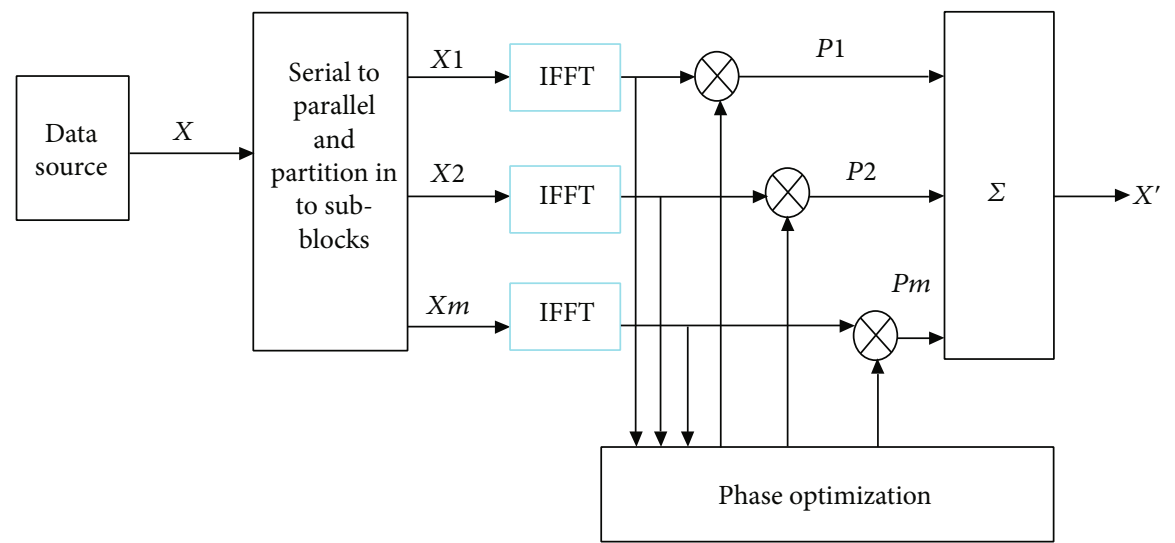

FIgURE 2: Block diagram of the conventional PTS scheme [23].

requires a high-speed and large dynamic range amplifier to forget perfect signal. These components are very expensive and lead to energy loss, shortening battery life in portable devices; besides, heat loss is not desirable in a fixed device [3].

One of the things to consider in the multicarrier transmission is the high-power ratio (PAPR) to normal signal transmission, as this is one of the OFDM signal negatives [4]. Therefore, this minimizes the range of the OFDM signal transmission. Furthermore, to avoid defects of the OFDM signal as a correction for burial between out-of-band radiation and subcarriers, the transmit power enhancer must be worked in its direct district. It is necessary to improve the transmission capacity in its immediate area. Therefore, the OFDM signal needs widespread backing, and this conversion is a waste of power [5]. OFDM is insensitive to the nonlinear effects of power amplifiers, due to the high peak-toaverage power ratio (PAPR), and this is one of the major problems in this system [6]. There are a lot of problems caused by high PAPR as it limits its wide use in some communications devices, and it is not preferred to use it in uplink transmission due to the restrictions in it [7]. It also leads to the consumption of large amounts of energy as the power amplifiers operate in the saturation region, and this leads to the deterioration of the bit error rate (BER) [8]. Also, high PAPR results in intermodulation and out-ofband radiation due to the nonlinearity in the power amplifier [9]. Therefore, many PAPR reduction schemes such as clipping and filtering $[10,11]$, block coding $[12,13]$, partial transmit sequence (PTS) [14], selected mapping (SLM) [15], tone injection (TI) [16], tone reservation (TR) [17], active constellation extension (ACE) [18], and random phase injection algorithms are used [19].

Despite the large number of methods used to reduce PAPR, where each method has pros and cons, there is still a problem of reducing complexity in the methods used, If 


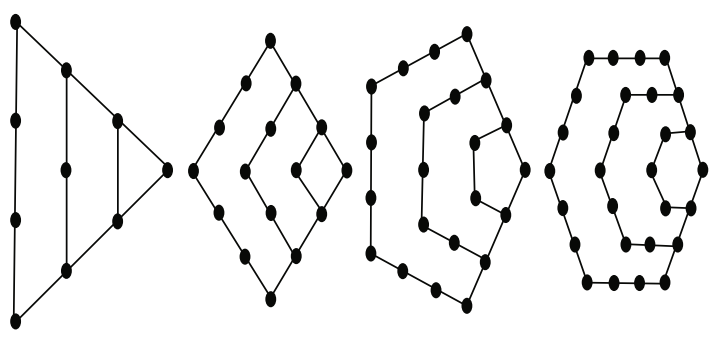

Figure 3: Type of polygon number: triangular, square, pentagonal, and hexagonal [25].

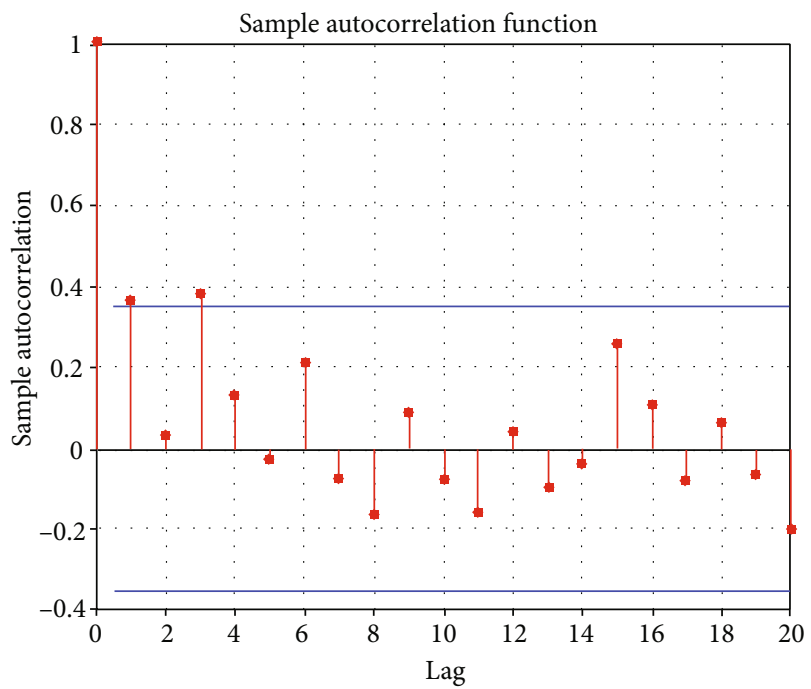

FIGURE 4: Input sequent numbers with different values.

we take, for example, the PTS and SLM methods, they have better performance in reducing PAPR and BER, but they have a problem with high computational complexity [20]. Among these theories that were used for reducing complexity in PTS is the 'rotating phase shift technique,' in which the signal was divided into groups, and this group was selected randomly. Moreover, choosing the low level of the PAPR depends on the different phase shifts according to a combination of factors [21]. The primary goal of source coding is to compress the original data intended for wireless transmission. Source coding techniques are used to greatly compress multimedia data [22]. A new method to reduce the PAPR value and complexity from the conventional PTS schemes is used. Polygon number is used to minimize the PAPR in the OFDM system, and good results are achieved when compared to the old method where the result was 4.7205 , where $N=64$ and no. of signal $=1000$. This method is one of the more recent methods used to improve OFDM defects. Moreover, when we compare the PN-PTS to the C-PTS or SLM, we can see how simple this algorithm is in comparison to the previous methods.

This paper is divided into seven sections. Section 1 outlines the introduction. Section 2 exposes the concept of the OFDM system and the peak-to-average power ratio (PAPR) and overviews the PAPR reduction scheme. Section 3 deals with the PTS scheme in the OFDM system, and Section 4

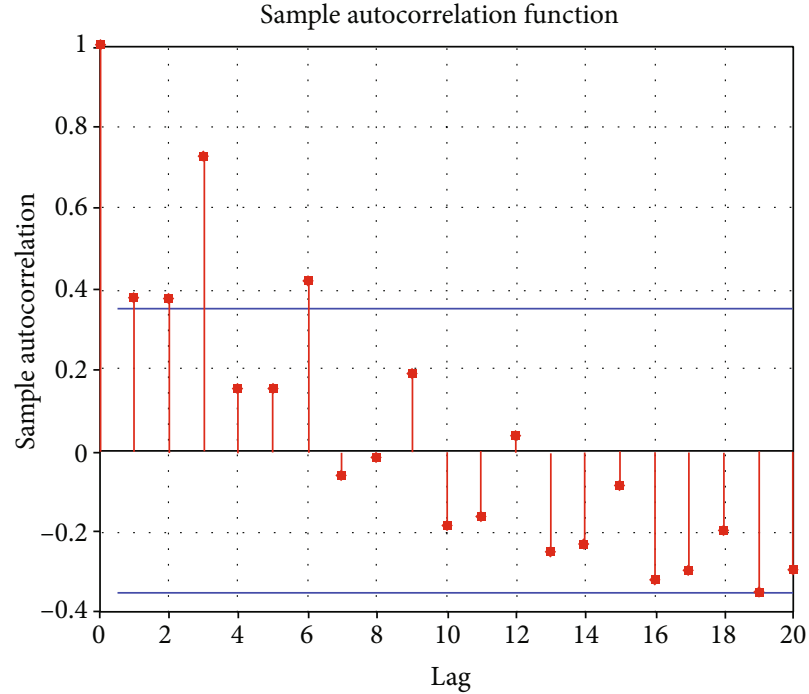

Figure 5: Processing number with autocorrelation.

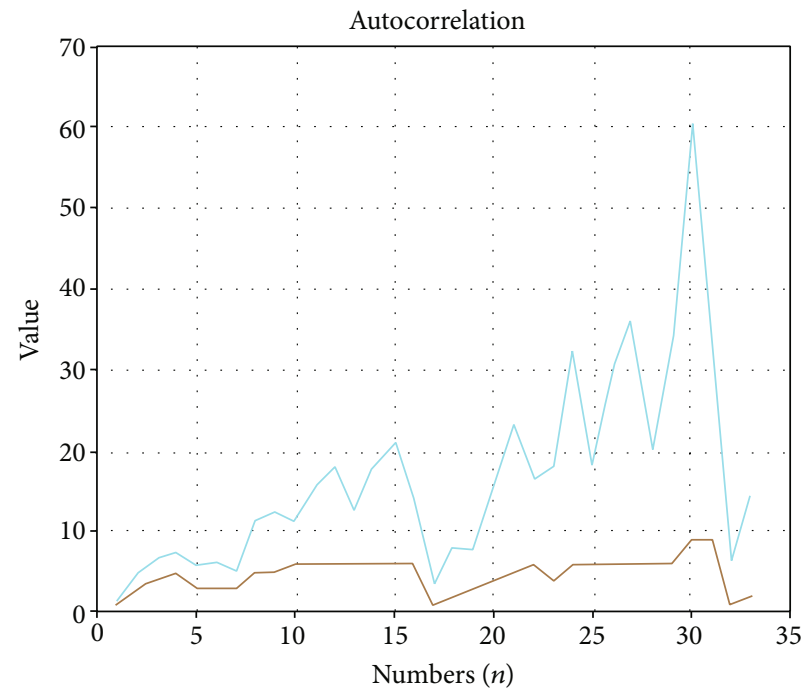

Data 1

- Data 2

Figure 6: Autocorrelation with different values.

presents the concept of the number theory, while in Section 5 the proposed PTS scheme is indicated, Section 6 discussed the simulation results, and the conclusion and future work are discussed in Section 7.

\section{OFDM System and PARP}

In an OFDM scheme, the data symbols used by the $N$ orthogonal subcarriers in the transmitter where $X=[X(0)$, $X(1) \cdots X(N-1)]$ time domain sequence are as shown in Figure 1. An OFDM signal can be generated and written as in (1) for IFFT:

$$
x(n)=\frac{1}{\sqrt{N}} \sum_{K=0}^{N-1} X_{K} e^{j \frac{2 \pi k n}{N}}, n=0,1 \cdots N-1 .
$$




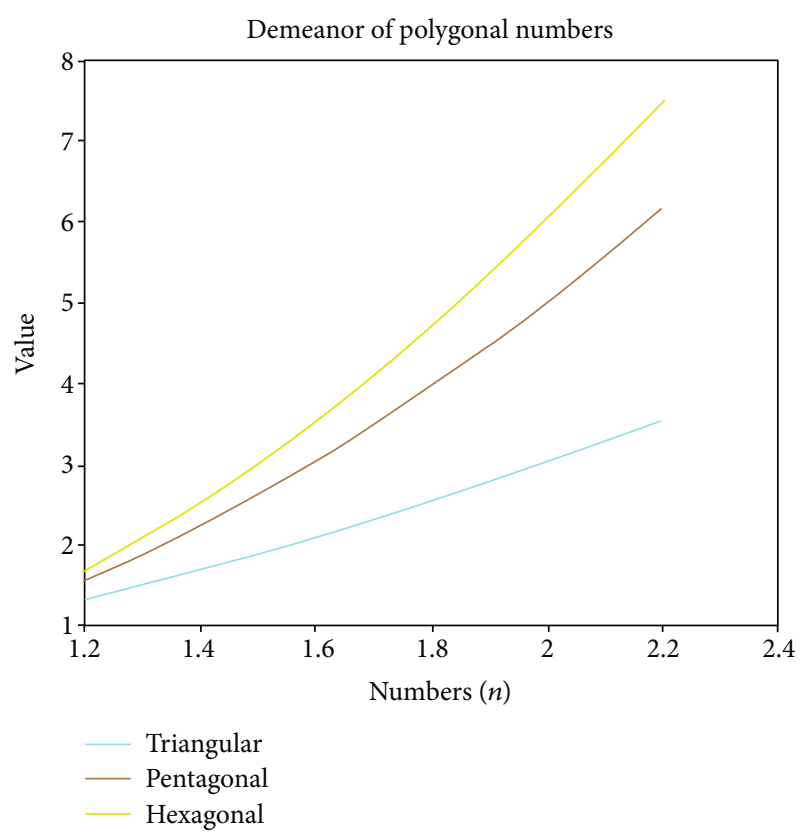

Figure 7: Demeanour of polygonal numbers for triangular, pentagonal, and hexagonal.

$X(n)$ is the discrete signal for the $N$ orthogonal subcarriers. This is in the time domain, so the OFDM symbol is often the variations in the envelope will be as of PAPR, where PAPR is the ratio of the actual signal power to the mean signal power [17].

The OFDM signal series $\mathrm{x}$ PAPR is named as

$$
\operatorname{PAPR}=\frac{\max _{0 \leq n<N|X(n)|^{2}}}{E\left[|X(n)|^{2}\right]},
$$

where $E[\cdot]$ stands for expectation. Complementary cumulative distribution function (CCDF) is used for the calculation value of PAPR level reduction performance in the OFDM system. PAPR CCDF can be described to given clip level PAPR as in (3) and (4).

$$
\mathrm{CDF} \mathrm{PAPR}=\operatorname{Pr}(\mathrm{PAPR}>\mathrm{PAPR} 0)
$$

$$
\mathrm{CCDFPAPR}=1-\left(1-e^{-\mathrm{PAPR} 0}\right)^{N},
$$

where $N$ is subcarrier numbers and PAPR0 is a certain threshold value.

\section{PTS Scheme in OFDM System}

In the conventional PTS scheme for the OFDM system, an input symbols vectors in the OFDM represent $X=$ $[X(0), \cdots . X(N-1)]^{T}$. The OFDM signal is generated by subcarriers $N$. The subcarrier generates a set of symbols. The subcarrier is separated from the adjacent subcarrier by

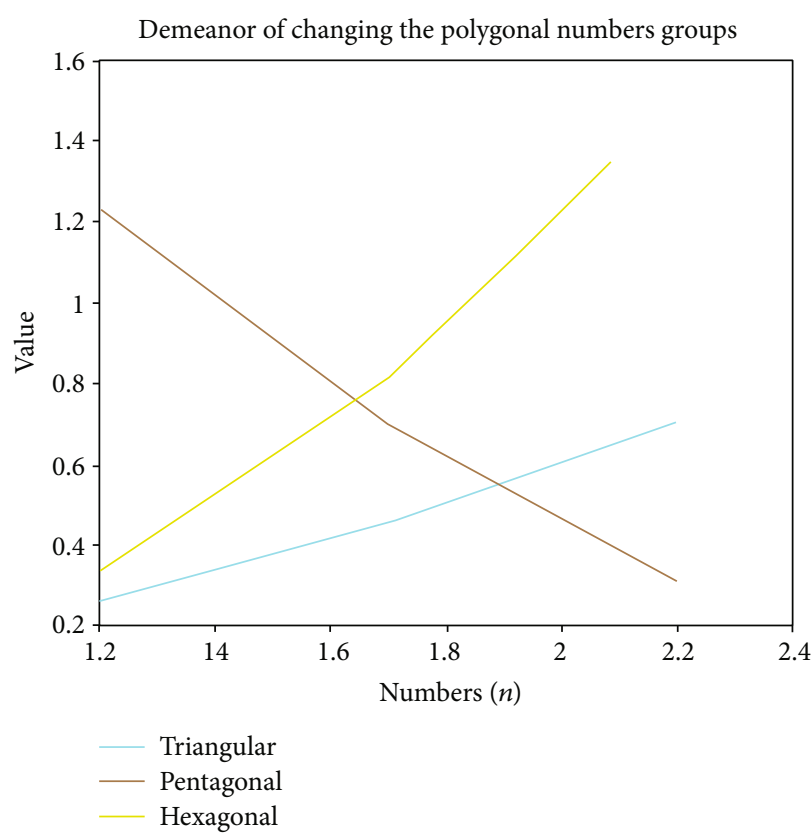

FIgURE 8: Demeanour of polygonal numbers when changing the value of a pentagonal number.

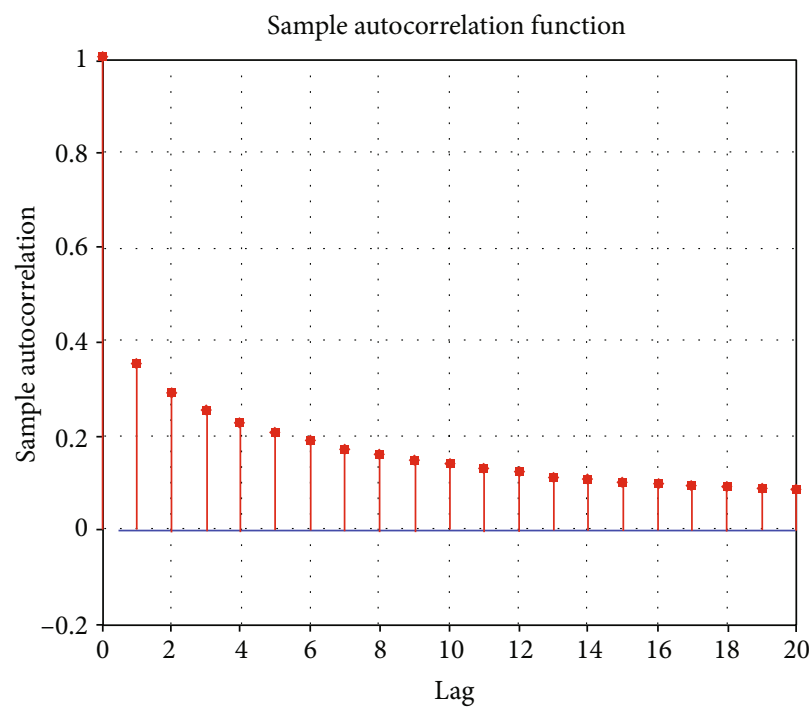

FIGURE 9: Data input with a range of magnitude 1.2-2.2.

the orthogonality of $N$ when $\Delta f=1 / T$, where $T$ is the signal duration of OFDM. The OFDM signal is expressed mathematically as follows:

$$
x[n]=\frac{1}{\sqrt{L N}} \sum_{i=0}^{L N-1} \bar{X}(l) e^{j 2 \pi l n / N}, 0 \leq n \leq L N-1 .
$$

$X$ here is a subcarrier vector for $N$ constellation symbols and $\varepsilon$ constellation, according to quadrature phase shift keying modulation (QPSK). The ratio of the maximum 


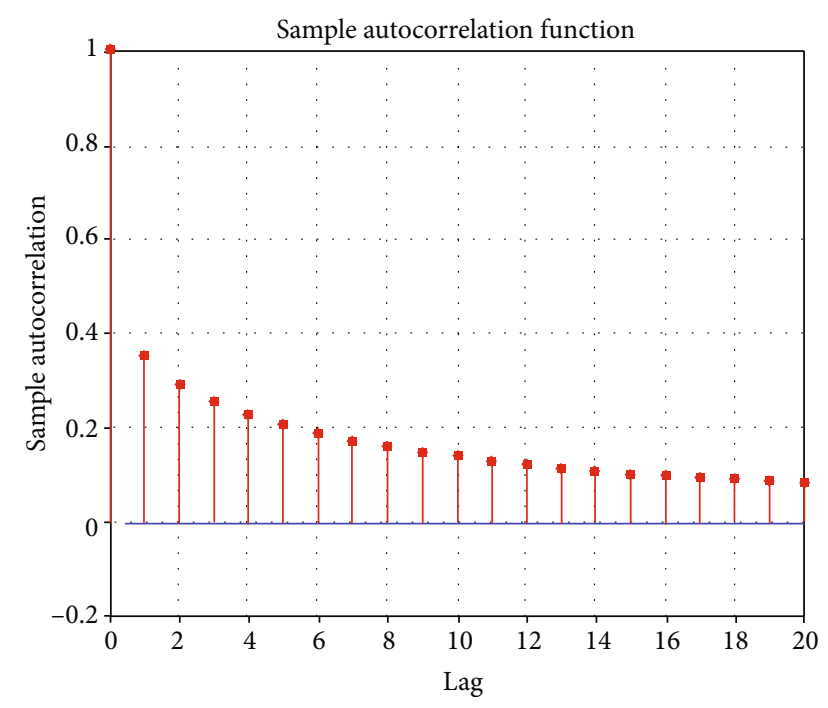

FIGURE 10: Data processing with a range of magnitude 1.2-2.2.

instantaneous power to the average power of the OFDM signal is known as the PAPR, and this can be expressed as in

$$
\mathrm{PAPR}=10 \log 10 \frac{\max |X(t)|^{2} 0 \leq t<T \mid}{P(a v)} .
$$

A sampling of the continuous-time signal $X(t)$ produces the separate transmitted signal $X[n]$ and often the Nyquist rate is taken; some information is lost at the height of $X(t)$ , and therefore, $X[n]$ is exceeded by a factor $L$ to obtain the signal $X(t)$ low value for PAPR. Factor $L$ helps to sequence $L N$-point data by inserting $N(L-1)$ zeros in the center of the $N$-point frequency domain signal and this is by $L N$ -point IFFT and this gives IFFT, which can be expressed as

$$
x[n]=\frac{1}{\sqrt{L N}} \sum_{i=0}^{L N-1} \bar{X}(l) e^{j 2 \pi l n / N}, 0 \leq n \leq L N-1,
$$

where

$$
[X(0), \cdots, X((N / 2)-1), \underbrace{0, \cdots, 0}_{(L-1) N}, X((N / 2)), \cdots, X\left(\mathrm{~N}_{-} 1\right)]^{T} \text {. It }
$$

shows that $L=4$ is enough to take the peak of the information $X(t)$.

The disjointed $M$ submasses are the result of dividing the input data block into $X$, and this relationship can be represented by vectors according to Equation (8), and this is in the PTS system to reduce PAPR in OFDM: $\left\{X_{m}, \mathrm{~m}=1,2\right.$.. , M]. Therefore, we can get

$$
X=\sum_{m=1}^{M} X_{m}
$$

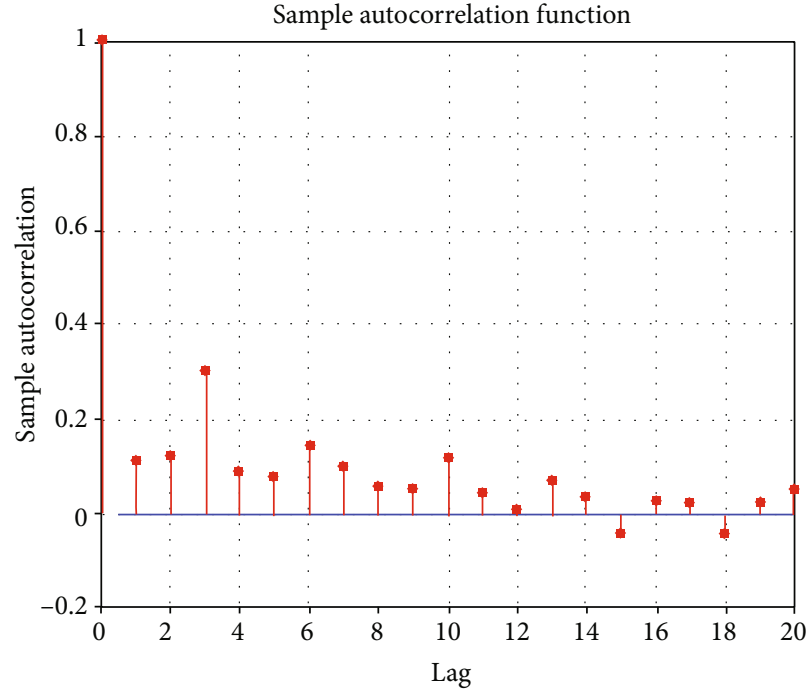

FIGURE 11: Data input with a range of magnitude 2.2-3.2.

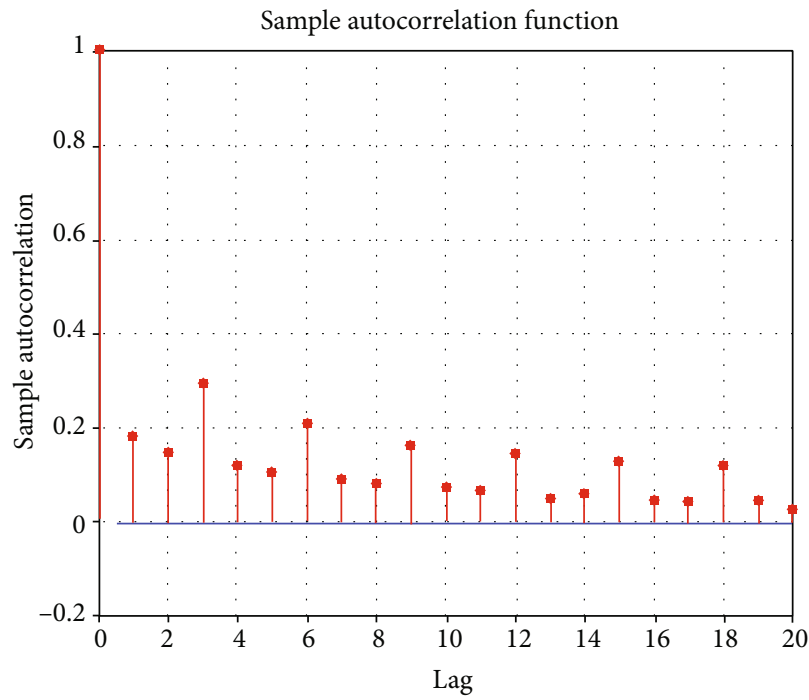

FIgURe 12: Data processing with a range of magnitude 2.2-3.2.

The sum of the subcarriers must be zero for the original signal which is displayed in another block. Then, the input symbol subblocks $X$ are transformed into $(M)$ timedomain partial transmit sequence. Therefore, $X$ can be represented as

$$
X_{m}=\operatorname{IFFT}_{L N \times N}\left\{X_{m}\right\}
$$

By incorporating phase factors $P=\left[P_{1}, P_{2}, \cdots P_{m}\right]$, this results in a group of candidates when PTS rotated independently. It can be represented as

$$
X=\sum_{m=1}^{M} P_{m X_{m}}=\sum_{m=1}^{M} \operatorname{IFFT}\left\{P_{m: X_{m}}\right\}
$$

It is possible to observe the special chart no. (4) for CPTS and to know that the choice of the candidate with the 


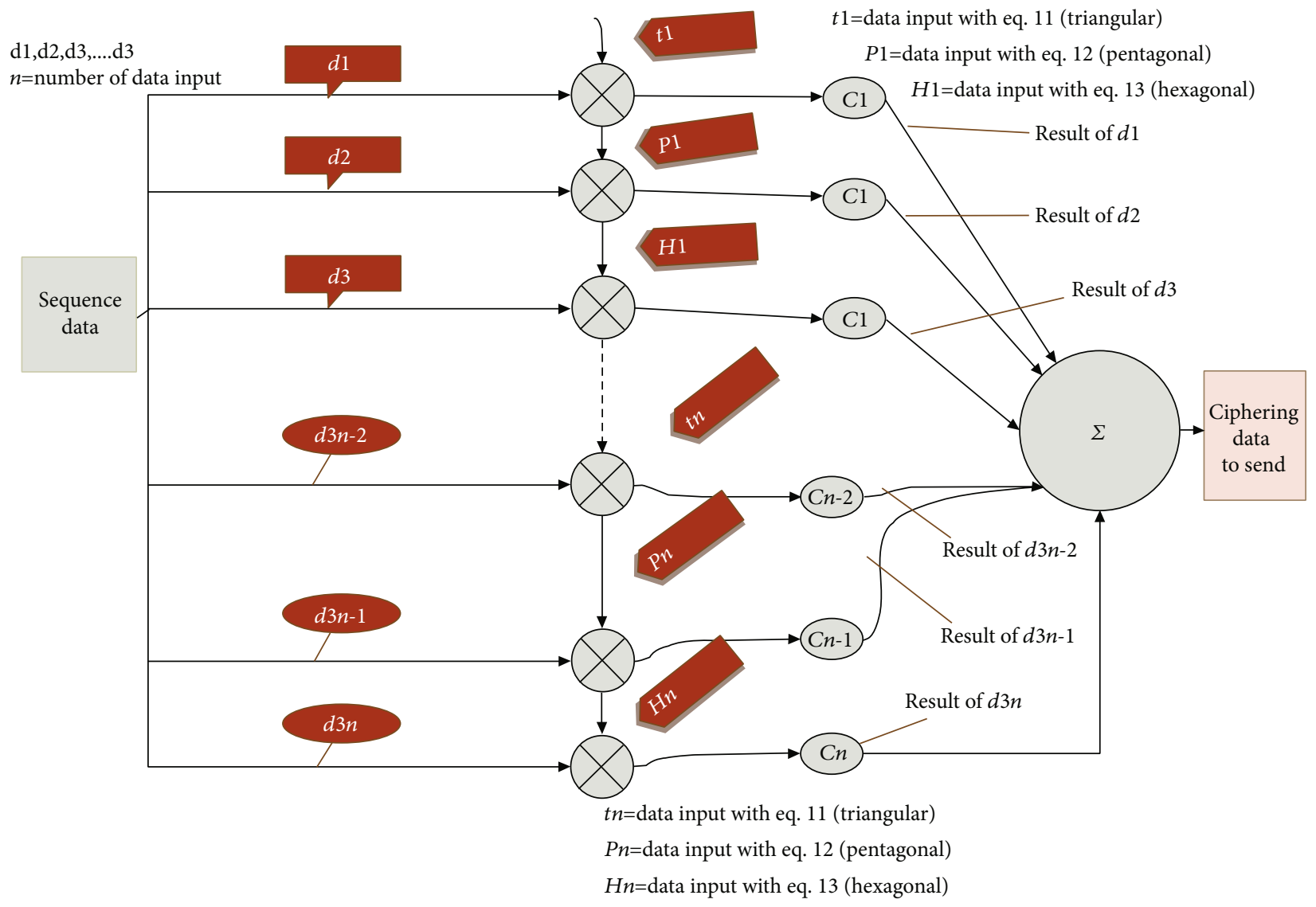

Figure 13: Principle of the polygonal numbers with data input and output to prevent similar data.

lowest PAPR is by searching the previous candidate for transmission, as to retrieve the original symbol $X$ by sending the side information bits to indicate the improved phase sequence and then to the receiver, as seen in Figure 2.

\section{Number Theory}

Number theory is a branch of pure mathematics mainly dedicated to studying the integral functions and integer. German mathematician Carl Friedrich Gauss (1777-1855) said: “ A Queen of Sciences is Arithmetic - and the Queen of Arithmetic is the theory of numbers" [24]. Polygon numbers are a type of number theory. The diagrams in Figure 3 show how polygon numbers are geometrically constructed. The illustrated polygonal numbers are, respectively, triangular, square, pentagonal, and hexagonal [25]. In the proposed algorithm, we took advantage of this theory in preventing data similarities and reducing the value of PAPR. According to this theory, the numbers of polygons are characterized by their preventing similarity, and this leads to sequence similarities being prevented. This feature makes us think about applying it to our work to reduce PAPR.

\section{Proposed PTS Scheme}

In this method, the geometric shape theory of numbers was used, and three sets of numbers 3, 5, and 6, for triangular, pentagonal, and hexagonal numbers, respectively, were used. The following are the stages that have been implemented in applying this algorithm to the conventional scheme.

5.1. Polygon Number Theory for Numbers 3, 5, and 6. Three types of groups have been taken, and these groups of numbers are not equal if $N$ is equal, as they depend on the geometry of the number. Below are the equations that were used in this proposed method in the form of each of these numbers:

$$
\begin{aligned}
t_{n} & =\frac{1}{2} n(n+1), \\
p_{n} & =\frac{1}{2} n(3 n+1), \\
h_{n} & =(2 n-1),
\end{aligned}
$$

where $t_{n}$ is a triangular number, $p_{n}$ refers to the pentagonal number, and $h_{n}$ is a hexagonal number.

We took advantage of this feature in the input data when sending it; as this feature is highlighted on the input data, it prevents similar data. For example, when three consecutive values come one after the other equal in value, and when multiplying each of these equal values by a number from the group chosen $(3,5$, and 6$)$, the result of these numbers will be unequal according to the succession property, as seen 


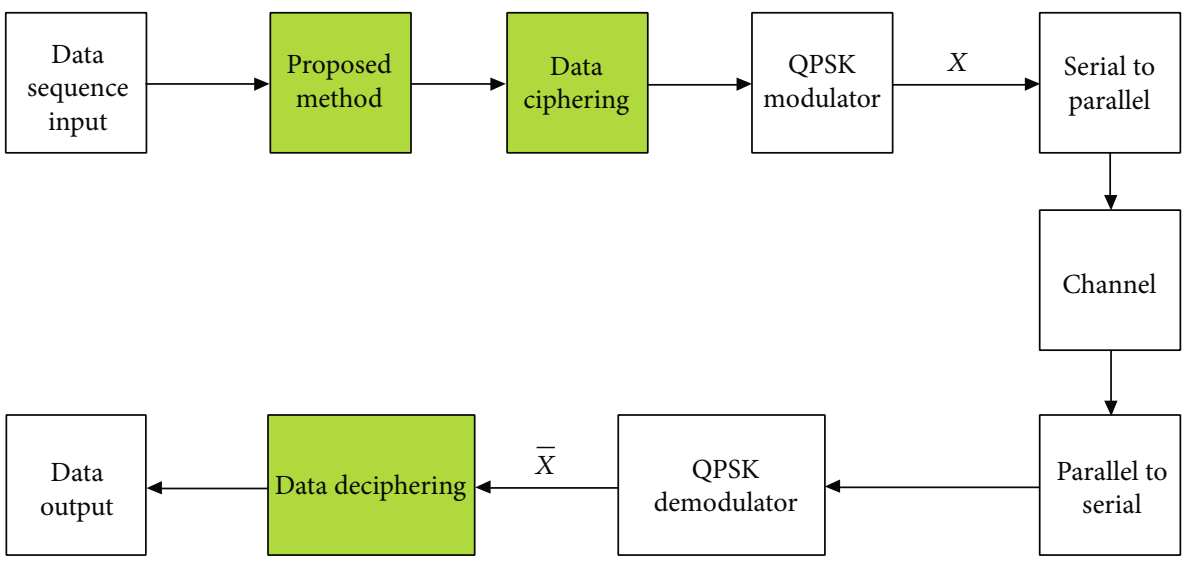

FIgURE 14: Principle block diagram of the proposed method.

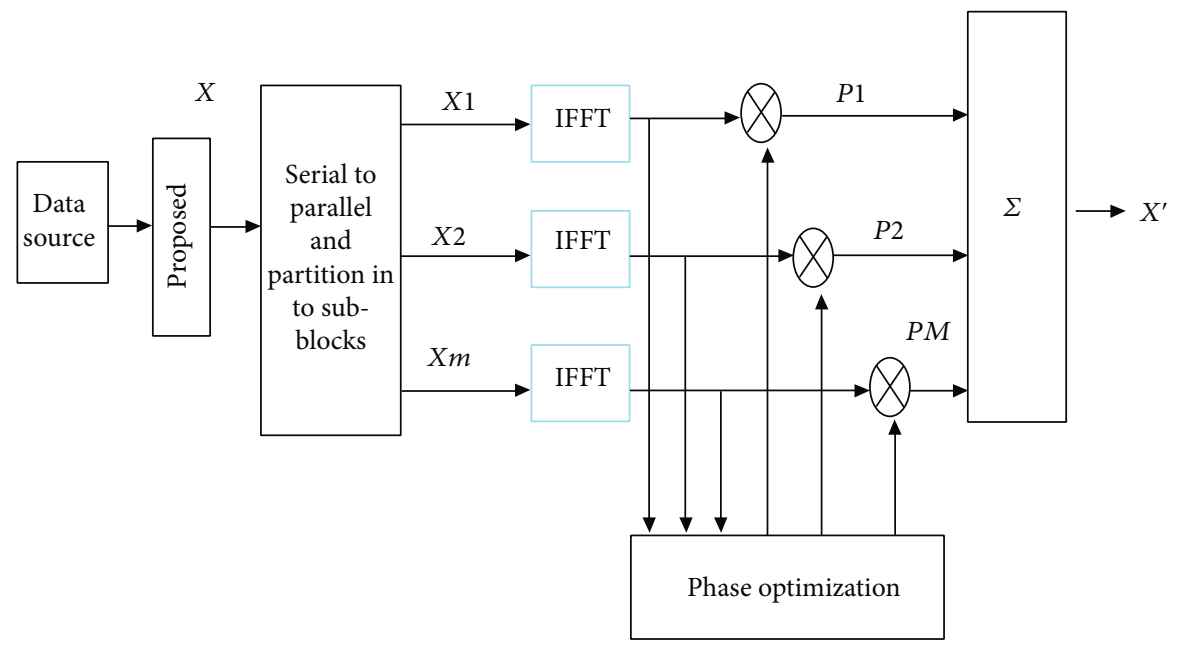

FIGURE 15: Block diagram of the proposed method with C-PTS scheme.

in Figures 4 and 5. This drawing, for the proposed method, shows the values that are not overlapping and preventing similar values from appearing. Moreover, Figure 6 depicts the amount of fluctuation that occurred due to similarities in numbers while they are progressing.

5.2. Effect of Theory. After knowing the effect of the theory on similar numbers, we made an experiment to analyse the effect on the shape of these three groups when increasing the value. This increase can be seen in Figure 7, whereas the amount of change that occurs when changing the value of each of these groups is shown in Figure 8.

5.3. Set of Periods. In this step, we made an experiment of a set of periods, which were divided into 10 values. After that, we chose a specific period from these periods, which is the selection of a portion of the abovementioned schemes, where we began to experiment with a period of (1.2-2.2) and another (2.2-3.2), etc. Meaning that the amount of the increase is 1 , and we noticed that there was no big difference when changing these values between one group and another. This confirms the increase in fluctuation that was mentioned in the previous clause, which was processed in the similarity of data. Figures 9-12 show these periods are taken.

These figures further demonstrate to us how to process similar data at different periods. Furthermore, it is clear from the figures that despite the fluctuation, it happens in all periods, but it differs in values. Therefore, to avoid amplifying the signal, we will choose one group over an average period for the rest of the work, which is (3.2-2.2).

5.4. Mechanism for the Proposed Method. After studying the effect of periods on data fluctuation, and to prevent amplification, the pentagonal number group was reversed (see Figures 9-12) to indicate the mechanism for the work of this algorithm by the proposed method. This can be observed in Figure 13, when the value of $N$ is equal, the method is based on the premise that the three terms are not equal, and when the value of $N$ is not equal, the algorithm is based on the progression property. According to Equations (11)-(13), the data do not overlap among them. We shed these data on the proposed algorithm, which can be observed in the sequence of this method as in Figure 14, where the data is entered on the algorithm, and then, it alternately changes 
TABLE 1: The parameters for the simulation.

\begin{tabular}{lc}
\hline Parameter & Value \\
\hline Number of OFDM signal & 10000 \\
Number of subcarriers $N$ & 64 \\
Modulation & 4,32 QPSK \\
IFFT \& FFT size & 256 \\
Oversampling factor $(L)$ & 4 \\
Number of partition p & 4
\end{tabular}

similar values to different values. The data generated is considered encrypted, and the resulting data is ready for the transmission process according to the conventional method steps in PTS shown in Figure 15. Upon receipt, decryption is performed to obtain the original data.

These figures show how to process similar data at different periods, and it is clear from the figures that despite the fluctuation, it happens in all periods, but it differs in values. Therefore, to avoid amplifying the signal, we choose one group over an average period for the rest of the work, which is $(3.2-2.2)$.

Similarly, Figure 15 is showing the proposed method that was applied to the (C-PTS) method, as the old method characteristics (mentioned before) used modified PTS to improve the PAPR. The first block is the input data source to the OFDM system, which is synthesized by subcarriers represented by a sequence of input codes in $X$ vector. The resultant is then converted into the $M$ group by subblocks, $X_{m}, 1 \leq m \leq M$.

Then, the time field signal is converted to IFFT multiplied by the phase factors; after that, we get the OFDM and then pick the best $X$ signal with the lowest PAPR point.

\section{Simulation Results}

In this research paper, we consider several simulation results to evaluate the performance in terms of PAPR reduction to demonstrate the effectiveness of the PN-PTS method. We used the MATLAB simulation program; the simulation results are based on the transmission of $10^{4}$ randomly generated OFDM symbols with $N=64$ carriers and an oversampling factor $L=4$. Table 1 lists the most important variables used in the simulation program to represents the PN-PTS method in which PTS was improved in reducing the PAPR.

The simulation results in Figure 16 show that when compared the PN-PTS with C-PTS, for 4QPSK, $N=32$, no.of OFDM signal $=1000$ symbols, and the original signal was $15.1263 \mathrm{~dB}$. It was processed in the C-PTS, and the amount of reduction was calculated $6.4068 \mathrm{~dB}$, The proposed scheme's (PN-PTS) PAPR performance is $4.6961 \mathrm{~dB}$.

In Figure 17, the following are seen: no.of OFDM signal $=10000$ symbols and the original signal was $15.1287 \mathrm{~dB}$. The PAPR reduction with C-PTS is $6.3157 \mathrm{~dB}$, and the PAPR performance for PN-PTS is $4.6826 \mathrm{~dB}$. This is only the first stage of minimization; the rest of the enhancements

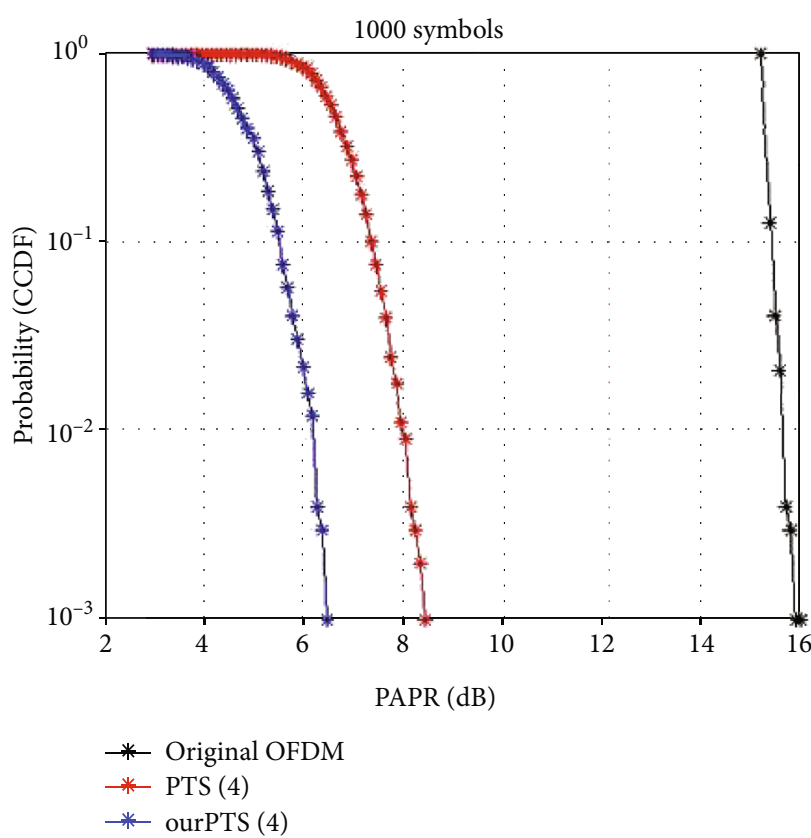

FIGURE 16: PAPR performance of the PN-PTS method with C-PTS for 4 QPSK with 1000 symbols.

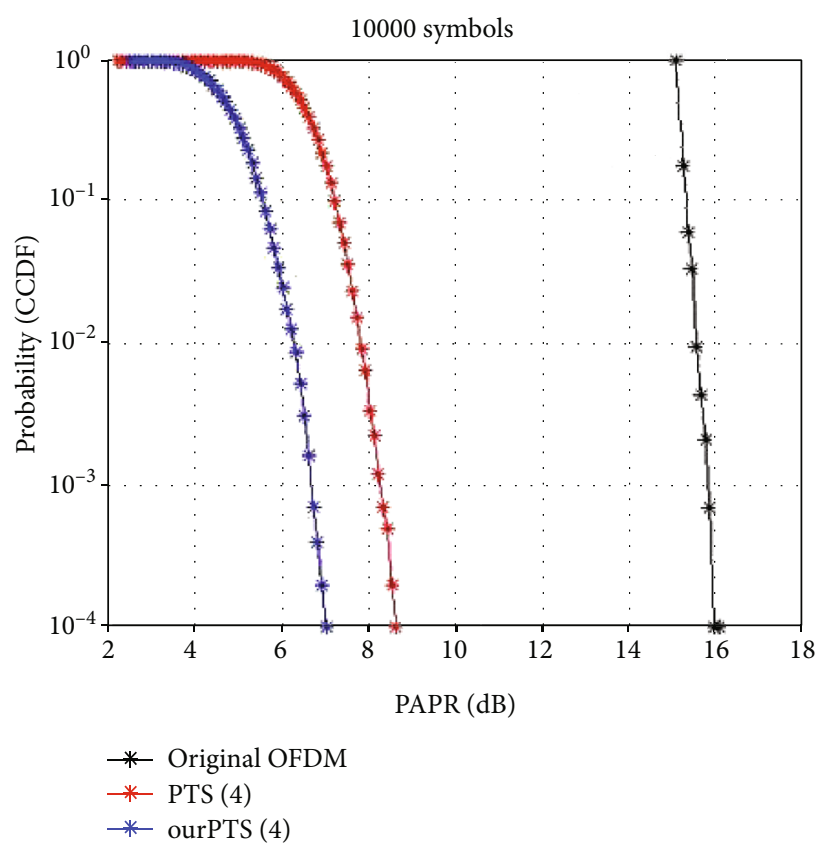

FIGURE 17: PAPR performance of the proposed scheme with C-PTS for 4 QPSK with 10000 symbols.

is shown in Table 2, which demonstrates the amount of progress made in reducing the PAPR.

In Figure 18 for simulation, 4 QPSK, the original signal is $15.1268 \mathrm{~dB}$, the no.of OFDM signal $=10000$ symbols, where the C-PTS gives $6.4376 \mathrm{~dB}$, while PAPR performance for PN-PTS gives $4.5683 \mathrm{~dB}$. In addition, when using 32 QPSK, the original signal is $17.9169 \mathrm{~dB}$, where we see the most improvement between the two methods is clear where 
TABLE 2: Computational complexity between the proposed and conventional schemes with a different number of subbands when $N=8,32$, and 64 .

\begin{tabular}{ccccc}
\hline$N$ & No. of OFDM signal & Original signal $(\mathrm{dB})$ & C-PTS & PN-PTS \\
\hline \multirow{2}{*}{8} & 1000 & 15.1263 & 6.4068 & 4.6961 \\
& 10000 & 15.1287 & 6.3157 & 4.6826 \\
\hline \multirow{2}{*}{32} & 1000 & 15.1342 & 6.4473 & 4.7059 \\
& 10000 & 15.1288 & 6.4169 & 4.7125 \\
\hline \multirow{2}{*}{64} & 1000 & 15.1268 & 6.4376 & 4.5683 \\
& 10000 & 15.1379 & 6.4503 & 4.7205 \\
\hline
\end{tabular}

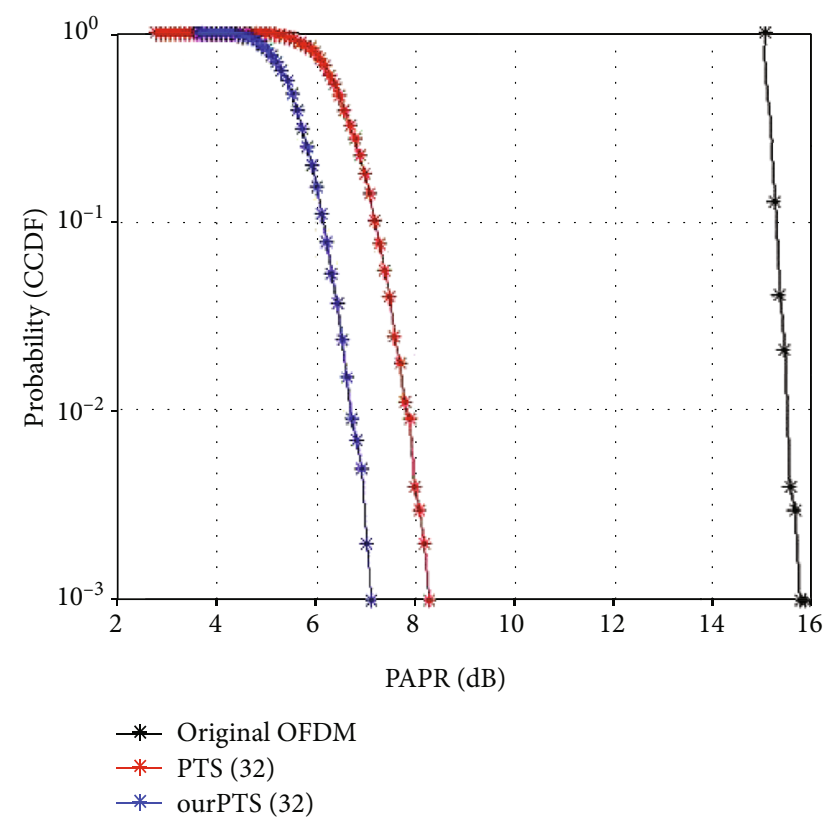

FIGURE 18: PAPR performance of the proposed scheme with CPTS, 32 QPSK.

TABle 3: Computational complexity between the proposed and conventional schemes with different modulations.

\begin{tabular}{lccc}
\hline QPSK modulation & Original signal $(\mathrm{dB})$ & C-PTS & PN-PTS \\
\hline 4 & 15.1268 & 6.4376 & 4.5683 \\
32 & 17.9169 & 6.4363 & 5.5072 \\
\hline
\end{tabular}

C-PTS gives worse PAPR performance $6.4363 \mathrm{~dB}$, while the PN-PTS gives $5.5072 \mathrm{~dB}$. Table 3 displays the results.

6.1. Discussion. When comparing the benefits of the proposed method to the conventional method and the previous methods that were used, we notice that this method is distinguished by an important feature, which has resulted in a reduction in the level of PAPR, which is the prevention of similar data when transmitting. In the conventional method, the selection of the candidate with the lowest PAPR is by searching for the previous candidate for transmission, which is accompanied by bits of the side information of the signal when retrieving the signal sent in the reception. The advan- tage of PN-PTS being simple to encrypt and simple to decrypt when returning data for transmission. Furthermore, it is less complexity.

Many methods have been used to reduce PAPR, including SLM, clipping, tone reservation, rotating phase shift, and hybrid methods, but the method we are used had never been used before. Simulation results have shown that the proposed scheme is reliable to estimate the selection of the PAPR.

\section{Conclusion}

In this paper, we proposed a PAPR reduction scheme for OFDM signals based on an improved PTS with polygon number algorithm (PN-PTS). To achieve the best PAPR performance possible, the proposed method is based on preventing similar data from being input during transmission. This has improved transmission by preventing the transmission of side information and lowering the increase in the PAPR. In addition, the PN-PTS algorithm has the advantage of being simple to encrypt and simple to decrypt when returning data for transmission. It is clear from the above results that using PN-PTS reduces PAPR. In addition, the amount of the reduction was good as the reduction between the proposed method and the conventional method was done by a good percentage from (6.4068 to 4.6961).

In the future, it is planned to apply this algorithm to other methods such as the 'rotating phase shift technique.'

\section{Data Availability}

Data sharing is not applicable to this article, as no new data were created or analysed in this study.

\section{Conflicts of Interest}

The authors declare that there is no conflict of interest regarding the publication of this paper.

\section{Acknowledgments}

The authors would like to thank Arab Open University and Oracle Saudi Arabia for supporting this study.

\section{References}

[1] K. Ramadan, M. I. Dessouky, and F. E. Abd El-Samie, "Modified OFDM configurations with equalization and CFO compensation for performance enhancement of OFDM communication systems using symmetry of the Fourier transform," AEU - International Journal of Electronics and Communications, vol. 126, article 153247, 2020.

[2] A. Khalil, N. Minallah, M. A. Awan, H. U. Khan, A. S. Khan, and A. U. Rehman, "On the performance of wireless video communication using iterative joint source channel decoding and transmitter diversity gain technique," Wireless Communications and Mobile Computing, vol. 2020, 16 pages, 2020.

[3] E. De Roux, M. Terosiet, F. Kölbl, M. Boissière, A. Histace, and O. Romain, "OFDM-based electrical impedance spectroscopy technique for pacemaker-induced fibrosis detection 
implemented in an ARM microprocessor," Microprocessors and Microsystems, vol. 70, pp. 38-46, 2019.

[4] A. A. Sharifi and M. Hosseinzadeh Aghdam, "A novel hybrid genetic algorithm to reduce the peak-to-average power ratio of OFDM signals," Computers and Electrical Engineering, vol. 80, article 106498, 2019.

[5] A. Kakkar, S. N. Garsha, O. Jain, and Kritika, "Improvisation in BER and PAPR by using hybrid reduction techniques in MIMO-OFDM employing channel estimation techniques," in 2017 IEEE 7th International Advance Computing Conference (IACC), pp. 170-173, Hyderabad, India, 2017.

[6] Y. C. Wang and Z. Q. Luo, "Optimized iterative clipping and filtering for PAPR reduction of OFDM signals," IEEE Transactions on Communications, vol. 59, no. 1, pp. 33-37, 2011.

[7] G. Wunder, R. F. H. Fischer, H. Boche, S. Litsyn, and J.-S. No, "The PAPR problem in OFDM transmission: new directions for a long-lasting problem," IEEE Signal Processing Magazine, vol. 30, no. 6, pp. 130-144, 2013.

[8] Y. Rahmatallah and S. Mohan, "Peak-to-average power ratio reduction in OFDM systems: a survey and taxonomy," IEEE Communication Surveys and Tutorials, vol. 15, no. 4, pp. 1567-1592, 2013.

[9] L. Yang, K. K. Soo, S. Q. Li, and Y. M. Siu, "PAPR reduction using low complexity PTS to construct of OFDM signals without side information," IEEE Transactions on Broadcasting, vol. 57, no. 2, pp. 284-290, 2011.

[10] X. Zhong, J. Qi, and J. Bao, "Using clipping and filtering algorithm to reduce PAPR of OFDM system," in 2011 International Conference on Electronics, Communications and Control (ICECC), pp. 1763-1766, Ningbo, China, 2011.

[11] S. Sengupta and B. K. Lande, "An approach to PAPR reduction in OFDM using Goppa codes," Procedia Computer Science, vol. 167, pp. 1268-1280, 2020.

[12] A. Idris, N. L. Mohd Sapari, M. Syarhan Idris, S. S. Sarnin, W. N. W. Mohamad, and N. F. Naim, "Reduction of PAPR using block coding method and APSK modulation techniques for F-OFDM in 5G system," in TENCON 2018 - 2018 IEEE Region 10 Conference, pp. 2456-2460, Jeju, Korea (South), 2019.

[13] H. A. Leftah, S. Boussakta, and S. Ikki, "Enhanced Alamouti space-time block-coding transmission based on a developed OFDM system," in 2014 9th International Symposium on Communication Systems, Networks \& Digital Sign (CSNDSP), pp. 591-595, Manchester, UK, 2014.

[14] Z. Zhou, L. Wang, and C. Hu, "Low-complexity PTS scheme for improving PAPR performance of OFDM systems," IEEE Access, vol. 7, pp. 131986-131994, 2019.

[15] V. Sudha and D. Sriram Kumar, "Low complexity PAPR reduction in SLM-OFDM system using time domain sequence separation," Alexandria Engineering Journal, vol. 57, no. 4, pp. 3111-3115, 2018.

[16] J. Tellado, Multicarrier Modulation with Low PAR: Applications to DSL and Wireless, vol. 587, Springer Science \& Business Media, 2006.

[17] J. Wang, X. Lv, and W. Wu, "SCR-based tone reservation schemes with fast convergence for PAPR reduction in OFDM system," IEEE Wireless Communications Letters, vol. 8, no. 2, pp. 624-627, 2019.

[18] Y. Xiao, L. Zhang, and M. Imran, "Active constellation extension for peak power reduction based on positive and negative iterations in OFDM systems," in 2019 UK/ China Emerging Technologies (UCET), pp. 1-5, Glasgow, UK, 2019.

[19] H. Nikookar and K. S. Lidsheim, "Random phase updating algorithm for OFDM transmission with low PAPR," IEEE Transactions on Broadcasting, vol. 48, no. 2, pp. 123-128, 2002.

[20] J. Ji, C. Zhang, and W. Zhu, "Low-complexity PTS scheme based on phase factor sequences optimization," Journal of Systems Engineering and Electronics, vol. 29, no. 4, pp. 707-713, 2018.

[21] A. K. Nahar, A. N. Abdalla, A. Y. Jaber, and M. M. Ezzaldean, "PAPR reduction using eight factors rotating phase shift technique based on local search algorithm in OFDM," Review of Computer Engineering Research, vol. 4, no. 2, pp. 38-53, 2017.

[22] N. Minallah, I. Ahmed, M. Ijaz, A. S. Khan, L. Hasan, and A. Rehman, "On the performance of self-concatenated coding for wireless mobile video transmission using DSTS-SP-assisted smart antenna system," Wireless Communications and Mobile Computing, vol. 2021, 10 pages, 2021.

[23] Y. A. Jawhar, L. Audah, M. A. Taher et al., "A review of partial transmit sequence for PAPR reduction in the OFDM systems," IEEE Access, vol. 7, pp. 18021-18041, 2019.

[24] T. Long Calvin, Elementary Introduction to Number Theory, vol. 77171950, Lexingt. DC Heath Company, LCCN, 1972.

[25] Gray, "Fermat's last theorem," in A History of Abstract Algebra: From Algebraic Equations to Modern Algebra, pp. 15-21, Cham: Springer International Publishing, 2018. 